\title{
International experimental fusion reactor-like extended Solovév equilibria with parallel flow
}

\author{
G. N. Throumoulopoulos ${ }^{1}, \mathrm{H}$. Tasso ${ }^{2}$ \\ 1 University of Ioannina, Association Euratom-Hellenic Republic, \\ Section of Theoretical Physics, GR 45110 Ioannina, Greece \\ ${ }^{2}$ Max-Planck-Institut für Plasmaphysik, Euratom Association, \\ D-85748 Garching, Germany
}

\begin{abstract}
A Solovév-like equilibrium solution is extended to plasmas with incompressible flow parallel to the magnetic field. ITER-like configurations are constructed for Alfvén Mach functions peaked either on or off the magnetic axis. The linear stability of the equilibrium is also examined by applying a sufficient condition.
\end{abstract}

Published in Phys. Plasmas 19, 014504 (2012). 
Since plasma flow is common in astrophysical systems and play a role in the transition to improved confinement regimes of magnetic confinement devices there has been an increasing interest to self consistent equilibria with flow [1]-[13]; they can be employed as starting points for stability and transport studies. In the static regime the most known and widely employed axisymmetric magnetohydrodynamic (MHD) analytic equilibrium is associated with the Solovév solution of the Grad-Sfafranov (GS) equation [14]. This solution has inherently a D-shaped separatrix with a couple of x-points on the high field side and a limited number of free parameters which puts a restriction on the construction of realistic configurations, particularly ITERlike ones with a single lower $\mathrm{x}$-point. This drawback was recently eliminated by an extension of the solution to contain arbitrary number of free parameters $[15,16]$. Consequently, a variety of equilibria were constructed with boundary shaping pertinent to laboratory fusion plasmas including both up-down symmetric and asymmetric configurations and desirable values of confinement figures of merit. Aim of the present contribution is to extend further this Solovév solution to plasmas with incompressible flow parallel to the magnetic field on the basis of a generalized GS equation [Eq. (1) below]. Then, ITER-like equilibria are constructed. A stability consideration is also made by means of a sufficient condition for linear stability [17].

We start from the generalized GS equation for field aligned incompressible flows,

$$
\left(1-M^{2}\right) \Delta^{\star} \psi-\frac{1}{2}\left(M^{2}\right)^{\prime}|\nabla \psi|^{2}+\frac{1}{2}\left(\frac{X^{2}}{1-M^{2}}\right)^{\prime}+\mu_{0} R^{2} P_{s}^{\prime}=0,
$$

together with the Bernoulli relation for the pressure,

$$
P=P_{s}(\psi)-\rho \frac{v^{2}}{2} .
$$

Here, $(z, R, \phi)$ are cylindrical coordinates with $z$ corresponding to the axis of symmetry; the function $\psi(R, z)$ labels the magnetic surfaces; $M(\psi)$ is the Mach function of the velocity with respect to the Alfvén velocity; $\rho(\psi)$ is the density and $X(\psi)$ relates to the toroidal magnetic field; for vanishing flow the surface function $P_{s}(\psi)$ coincides with the pressure; $v$ is the velocity modulus which can be expressed in terms of surface functions and $R ; \Delta^{\star}=R^{2} \nabla$. $\left(\nabla / R^{2}\right)$; and the prime denotes a derivative with respect to $\psi$. Since the flow contribution to the pressure in (2) is always non-negative, the requirement 
$P \geq 0$ sets certain limit on the maximum permissible flow. Derivation of (1) and (2) in the general case of flows non-parallel to the magnetic field is provided in Ref. [4]. Eq. (1) can be simplified by the transformation

$$
u(\psi)=\int_{0}^{\psi}\left[1-M^{2}(g)\right]^{1 / 2} d g
$$

which reduces (1) to

$$
\Delta^{\star} u+\frac{1}{2} \frac{d}{d u}\left(\frac{X^{2}}{1-M^{2}}\right)+\mu_{0} R^{2} \frac{d P_{s}}{d u}=0 .
$$

Transformation (3) does not affect the magnetic surfaces, it just relabels them. Note that no quadratic term as $|\nabla u|^{2}$ appears anymore in (4) and this equation becomes identical in form with the usual (static) GS equation. The surface quantities $X^{2} /\left(1-M^{2}\right)$ and $P_{s}(\psi)$ are free functions for each choice of which (4) is fully determined and can be solved whence the boundary condition for $u$ is given. Therefore, any analytic solution to the GS equation can be smoothly extended to the parallel flow case. For convenience we will introduce dimensionless quantities by $\tilde{R}=R / R_{0}, \tilde{z}=z / R_{0}, \tilde{u}=u /\left(B_{0} R_{0}^{2}\right)$, $\tilde{\rho}=\rho / \rho_{0}, \tilde{P}=P /\left(B_{0}^{2} / \mu_{0}\right) ; \tilde{\mathbf{j}}=\mathbf{j} /\left(B_{0} /\left(\mu_{0} R_{0}\right)\right)$, where $\mathbf{j}$ is the current density, and $\tilde{\mathbf{v}}=\mathbf{v} / v_{A 0}$, where $v_{A 0}=B_{0} / \sqrt{\mu_{0} \rho_{0}}$. The free parameters $R_{0}$ and $B_{0}$ are the radial coordinate of the geometric center of the configuration and the vacuum toroidal magnetic field thereon. Then, Eq. (4) remains identical in form for the tilted quantities by formally setting $\mu_{0}=1$. In the following the tilde will be dropped on the understanding of non-dimensionality.

By making the Solovév-like linearizing ansatz $P_{s}=P_{1} u$ and $X^{2} /(1-$ $\left.M^{2}\right)=X_{0}^{2}+2 X_{1} u$, the resulting form of Eq. (4) admits the following solution which consists the basis of the present study:

$$
\begin{gathered}
u=u_{h}+u_{p}, \\
u_{h}=c_{1}+c_{2} \frac{R^{2}}{2}+c_{3}\left(z^{2}+\frac{R^{2}}{2}-R^{2} \ln R\right)+c_{4}\left(\frac{z^{2} R^{2}}{2}-\frac{R^{4}}{8}\right) \\
+c_{5}\left(z^{4}+3 z^{2} R^{2}-\frac{15 R^{4}}{8}-6 z^{2} R^{2} \ln R+\frac{3}{2} R^{4} \ln R\right) \\
+c_{6}\left(\frac{z^{4} R^{2}}{2}-\frac{3 z^{2} R^{4}}{4}+\frac{R^{6}}{16}\right)
\end{gathered}
$$




$$
\begin{aligned}
& +c_{7}\left(z^{6}+\frac{15 z^{4} R^{2}}{2}-\frac{255 z^{2} R^{4}}{8}+\frac{25 R^{6}}{8}-15 z^{4} R^{2} \ln R\right. \\
& \left.+\frac{45}{2} z^{2} R^{4} \ln R-\frac{15}{8} R^{6} \ln R\right) \\
& +d_{1} z+d_{2} \frac{z R^{2}}{2}+d_{3}\left(z^{3}+\frac{3 z R^{2}}{2}-3 z R^{2} \ln R\right)+d_{4}\left(\frac{z^{3} R^{2}}{2}-\frac{3 z R^{4}}{8}\right) \\
& +d_{5}\left(z^{5}+5 z^{3} R^{2}-\frac{75 z R^{4}}{8}-10 z^{3} R^{2} \ln R+\frac{15}{2} z R^{4} \ln R\right) \\
& u_{p}=\frac{P_{1}}{8} R^{4}-\frac{X_{1}}{2} z^{2} .
\end{aligned}
$$

Here, $u_{h}$ is the solution of the homogeneous equation and $u_{p}$ is a particular solution of the inhomogeneous equation. $u_{h}$ consists of a symmetric in $z$ part in connection with the coefficients $c_{i}$ and an asymmetric in $z$ part in connection with the coefficients $d_{j}$. The construction of this solution is based on an iterative algorithm which is explained in Sec. 2 of Ref. [16]. This algorithm permits arbitrary number of symmetric and asymmetric terms in the homogeneous solution and therefore arbitrary number of free parameters which can be specified in connection with desirable boundary shaping and confinement figures of merit. For $d_{j}=0$ the equilibrium is up-down symmetric while an ITER-like equilibrium requires non symmetric terms. Here we have chosen seven symmetric and five asymmetric terms and therefore there are 14 free parameters including $P_{1}$ and $X_{1}$. Note that $X_{0}=1$ because of the adopted normalization. It is emphasized that (5)-(7) hold for arbitrary Mach functions and densities.

By exploiting the free parameters we have constructed ITER-like configurations with the following characteristics: major radius $R_{0}=6.2 \mathrm{~m}$, minor radius $a=2 m$, elongation $\kappa=1.33$, triangularity $\delta=0.33$, safety factor on axis in the interval $1 \leq q_{a} \leq 2$ and average toroidal beta $\beta_{t} \approx 0.01$. The parametric values were fixed, similar to Ref. [15], by solving numerically the set of equations (8)-(14), (17) and (18) to follow. First, without prescribing completely the boundary curve we impose $u$ to vanish at four characteristic fixed points of the boundary, i.e. the inner point, $\left(R_{i n}, z=0\right)$, the outer point, $\left(R_{\text {out }}, z=0\right)$, the higher point, $\left(R_{u}, z_{u}\right)$, and the (lower) x-point, $\left(R_{x}, z_{x}\right)$ :

$$
u\left(R_{\text {in }}, 0\right)=u\left(R_{\text {out }}, 0\right)=u\left(R_{u}, z_{u}\right)=u\left(R_{x}, z_{x}\right)=0 .
$$


At the higher point and the $x$-point should hold the relations

$$
\begin{gathered}
\frac{\partial u}{\partial R}\left(R_{u}, z_{u}\right)=0 \\
\frac{\partial u}{\partial R}\left(R_{x}, z_{x}\right)=\frac{\partial u}{\partial z}\left(R_{x}, z_{x}\right)=0 .
\end{gathered}
$$

Also, we require that the configuration is up-down symmetric near the plane $z=0$ :

$$
\frac{\partial u}{\partial z}\left(R_{\text {in }}, 0\right)=\frac{\partial u}{\partial z}\left(R_{\text {out }}, 0\right)=0 .
$$

Furthermore, it can be shown that the curvature of the bounding curve at the inner, outer and higher points should satisfy the relations [15]

$$
\begin{aligned}
\frac{\partial^{2} u}{\partial z^{2}}\left(R_{\text {in }}, 0\right) & =-N_{1} \frac{\partial u}{\partial R}\left(R_{\text {in }}, 0\right), \\
\frac{\partial^{2} u}{\partial z^{2}}\left(R_{\text {out }}, 0\right) & =-N_{2} \frac{\partial u}{\partial R}\left(R_{\text {out }}, 0\right), \\
\frac{\partial^{2} u}{\partial R^{2}}\left(R_{u}, z_{u}\right) & =-N_{3} \frac{\partial u}{\partial z}\left(R_{u}, z_{u}\right),
\end{aligned}
$$

where

$$
\begin{aligned}
N_{1} & =\frac{(1-\alpha)^{2}}{\epsilon \kappa^{2}}, \\
N_{2} & =-\frac{(1+\alpha)^{2}}{\epsilon \kappa^{2}}, \\
N_{3} & =-\frac{\kappa}{\epsilon} \cos \alpha^{2}, \\
\alpha & =\arcsin \delta .
\end{aligned}
$$

Derivation of (12)-(14) is based on the relation $d u=(\partial u / \partial R) d R+(\partial u / \partial z) d z$ from which on the poloidal cross-section of a magnetic surface can be written as

$$
\frac{\partial u}{\partial z}=-\left.\frac{d R}{d z}\right|_{u} \frac{\partial u}{\partial R}
$$

Partial derivation of (15) with respect to $z$, on account of $d R(z=0) /\left.d z\right|_{u}=0$, leads for $z=0$ to

$$
\frac{\partial^{2} u}{\partial z^{2}}=-\left.\frac{d^{2} R}{d z^{2}}\right|_{u} \frac{\partial u}{\partial R}
$$


The term $d^{2} R /\left.d z^{2}\right|_{u}$ in (16) can be calculated by employing for the upper, D-shaped part of the boundary the parametric equations

$$
\begin{aligned}
R & =1+\epsilon \cos (\tau+\alpha \sin \tau) \\
z & =\epsilon \kappa \sin \tau
\end{aligned}
$$

where $\tau$ is a parameter covering the range $0 \leq \tau \leq \pi, \epsilon=a / R$ is the inverse aspect ratio, $\kappa$ is the elongation and $\sin \alpha=\delta$ is the triangularity. Application of (16) for $R_{\text {in }}$ and $R_{\text {out }}$ furnishes relations (12) and (13). Relation (14) for the upper point can be derived in a similar way.

In addition to the above boundary shaping equations we employ for the safety factor on axis and the toroidal beta the relations:

$$
q_{a}=\left.\frac{X}{R \sqrt{1-M^{2}}}\left(\frac{\partial^{2} u}{\partial R^{2}} \frac{\partial^{2} u}{\partial z^{2}}\right)^{-1 / 2}\right|_{R=R_{a}, z=z_{a}},
$$

where $\left(R_{a}, z_{a}\right)$ is the position of the magnetic axis, and

$$
\beta_{t}=\frac{\int_{V} P d \tau}{B_{0}^{2} /\left(2 \mu_{0}\right)}
$$

Regarding the flow, we adopted the following alternative choices of $M^{2}$ :

$$
\begin{aligned}
& M^{2}=M_{a}^{2}\left(\frac{u}{u_{a}}\right)^{n} \\
& M^{2}=C u^{n}\left(u_{a}-u\right)^{m}
\end{aligned}
$$

where

$$
C=M_{a}^{2}\left[\frac{m u_{a}}{m+n}\right]^{-m}\left[\frac{n u_{a}}{m+n}\right]^{-n} .
$$

Here, $u_{a}$ refers to the magnetic axis, the free parameter $M_{a}^{2}$ corresponds to the maximum value of $M^{2}$ and $m$ and $n$ are related to the flow shear. In particular, (19) is peaked on- while (20) is peaked off-axis in connection with respective auxiliary heating of tokamaks. Typical value of $M^{2}$ for (large) tokamaks are of the order of $10^{-4}$ because of the experimental scaling $v \sim$ $10^{-1} v_{s}$, where $v_{s}=(\gamma P / \rho)^{1 / 2}$ is the sound velocity. An example of the equilibria constructed is shown in Fig. 1.

We now consider the important issue of the stability of (5)-(7), with respect to small linear MHD perturbations by applying a sufficient condition 


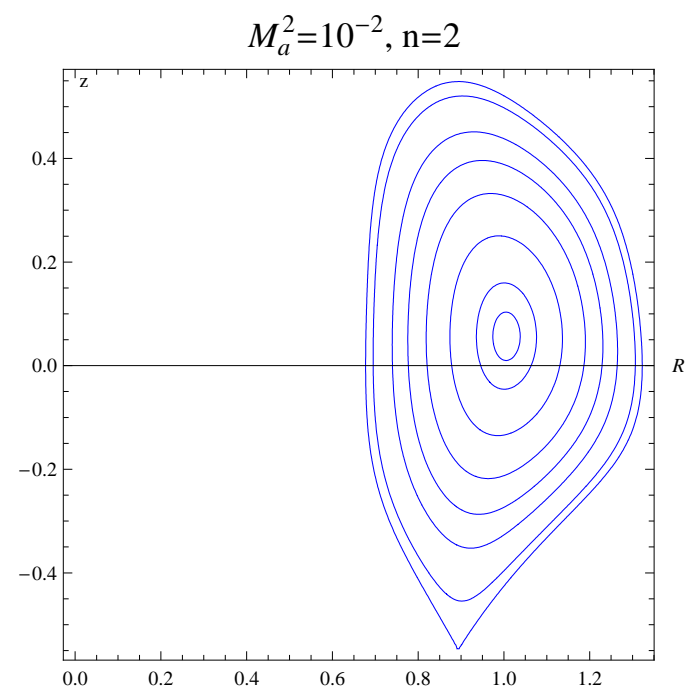

Figure 1: An ITER-like equilibrium with parallel plasma flow in connection with solution (5)-(7) with $q_{a}=1.1$ and $\beta_{t}=0.01$.

(Ref. [17]). This condition states that a general steady state of a plasma of constant density and incompressible flow parallel to $\mathbf{B}$ is linearly stable to small three-dimensional perturbations if the flow is sub-Alfvénic $\left(M^{2}<1\right)$ and $A \geq 0$, where $A$ is given below by (21)-(26). Consequently, we set $\rho=1$. In fact if the density is uniform at equilibrium it remains so at the perturbed state because of incompressibility [18]. In the $u$-space for axisymmetric equilibria, $A$ assumes the form

$$
\begin{aligned}
A & =A_{1}+A_{2}+A_{3}+A_{4}, \\
A_{1} & =-(\mathbf{j} \times \nabla u)^{2}, \\
A_{2} & =(\mathbf{j} \times \nabla u) \cdot(\boldsymbol{\nabla} u \cdot \nabla) \mathbf{B}, \\
A_{3} & =-\frac{1}{2} \frac{d M^{2}}{d u}\left(1-M^{2}\right)^{-1}|\nabla u|^{2} \nabla u \cdot \frac{\boldsymbol{\nabla} B^{2}}{2}, \\
A_{4} & =-\frac{1}{2} \frac{d M^{2}}{d u}\left(1-M^{2}\right)^{-3 / 2}|\nabla u|^{4} g,
\end{aligned}
$$




$$
g=\left(1-M^{2}\right)^{-1 / 2}\left(\frac{d P_{\mathrm{s}}}{d u}-\frac{d M^{2}}{d u} \frac{B^{2}}{2}\right)
$$

The quantity $A_{1}$ is a destabilizing contribution $\left(A_{1}<0\right)$ potentially related

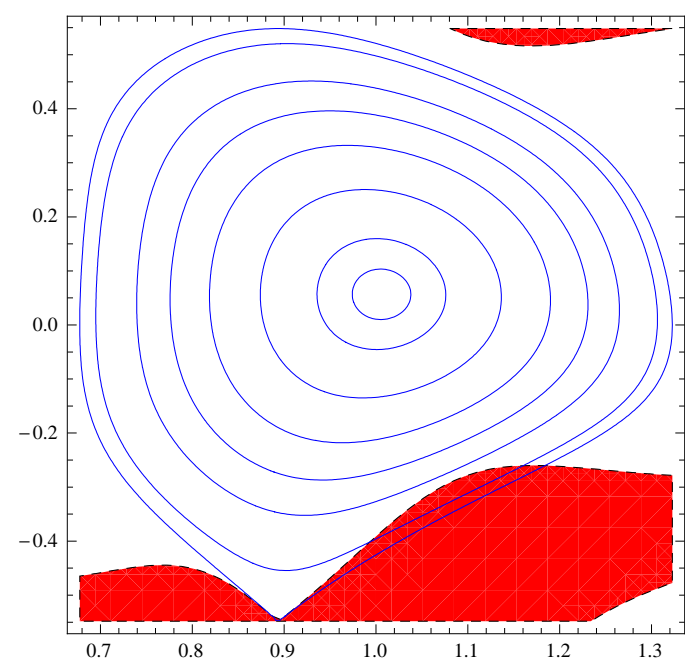

Figure 2: Stability diagram showing the sign of the quantity $A$ for the Mach function (19) with $M_{a}^{2}=10^{-2}$. In the red colored region (gray shaded) regions where $A \geq 0$ the stability condition is satisfied on the understanding that the physically relevant part lies within the plasma boundary.

to current driven modes while $A_{2}$ relates to the variation of the magnetic field perpendicular to the magnetic surfaces. $A_{3}$ and $A_{4}$ are flow terms depending on the magnitude and the shear of the flow (in connection with the parameters $M_{a}^{2}, n$ and $m$ for the present study). The quantity $A$ was calculated analytically by Mathematica. It turns out that the condition $A \geq 0$ is satisfied only in a small region close to the boundary irrespective of flow. As an example, the stability diagram showing the sign of $A$ on the poloidal plane is given in Fig. 2 for the Mach-function (19) and the nearly maximum permissible value $M_{a}^{2}=10^{-2}$ (in connection with the non-negativeness of the pressure). The condition is satisfied in the red colored (gray shaded) region. The diagram is nearly identical with the respective static one, i.e. $\left|\Delta A / A_{q s}\right| \leq 10^{-3}$, where $A_{q s}$ are the static values of $A$ and $\Delta A$ their differences from the stationary ones. Also, a similar result holds for the off-axis Mach function (20). Stability diagrams for the terms $A_{1}, A_{2}, A_{3}$ and $A_{4}$ in 
$A_{2}$

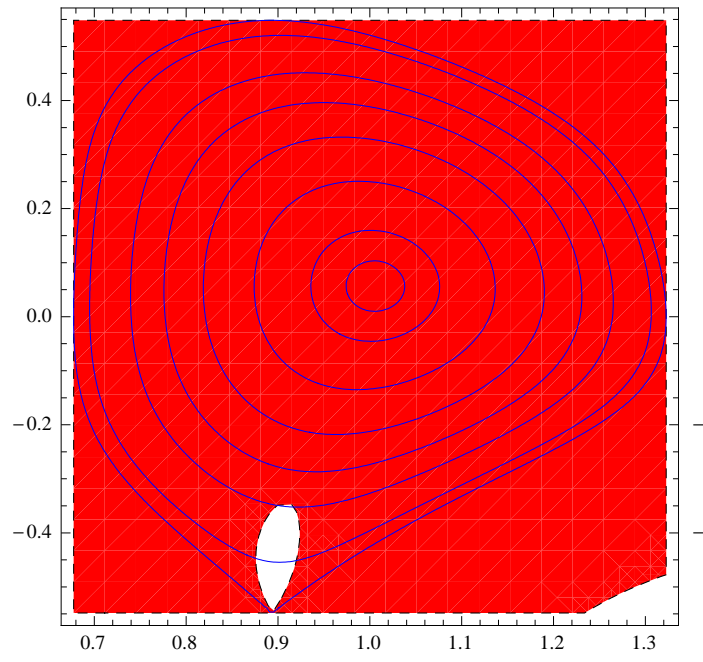

$A_{3}$

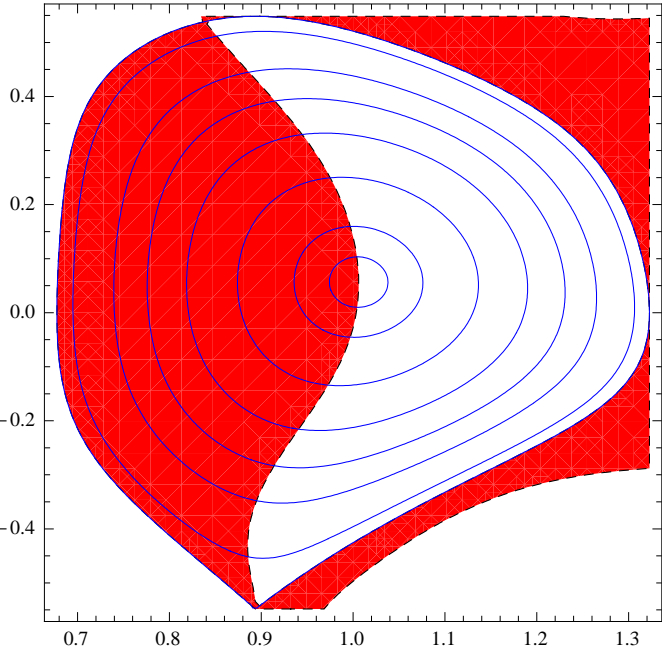

$A_{4}$

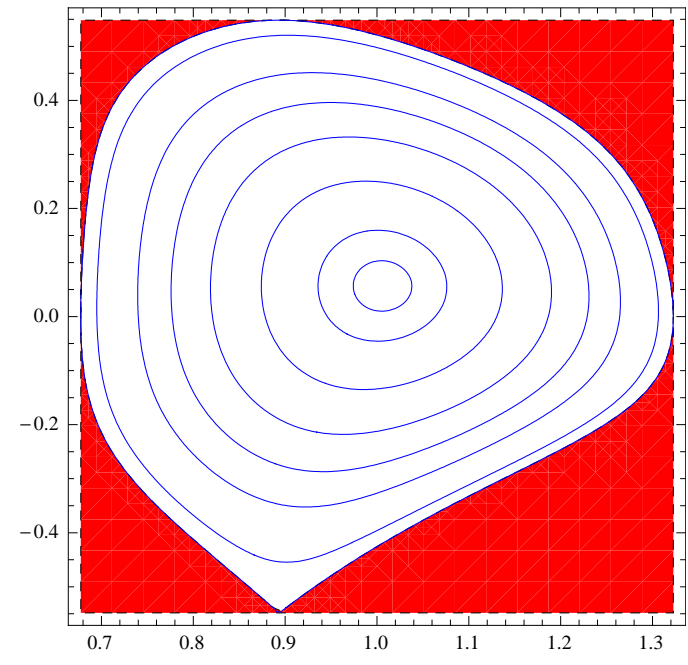

Figure 3: Stability diagrams for the terms $A_{2}, A_{3}$ and $A_{4}$ of the stability quantity $A$ for the Mach function (19) with $M_{a}^{2}=10^{-2}$. In the red colored (gray shaded) regions these terms are non-negative.

Fig. 3 show that $A_{2}$ is nearly everywhere stabilizing and of the same order of magnitude as $A_{1}$ but not large enough to overcome the destabilizing effect of $A_{1}$ (Fig. 4). The flow term $A_{3}$ is stabilizing over a large part of the plasma 


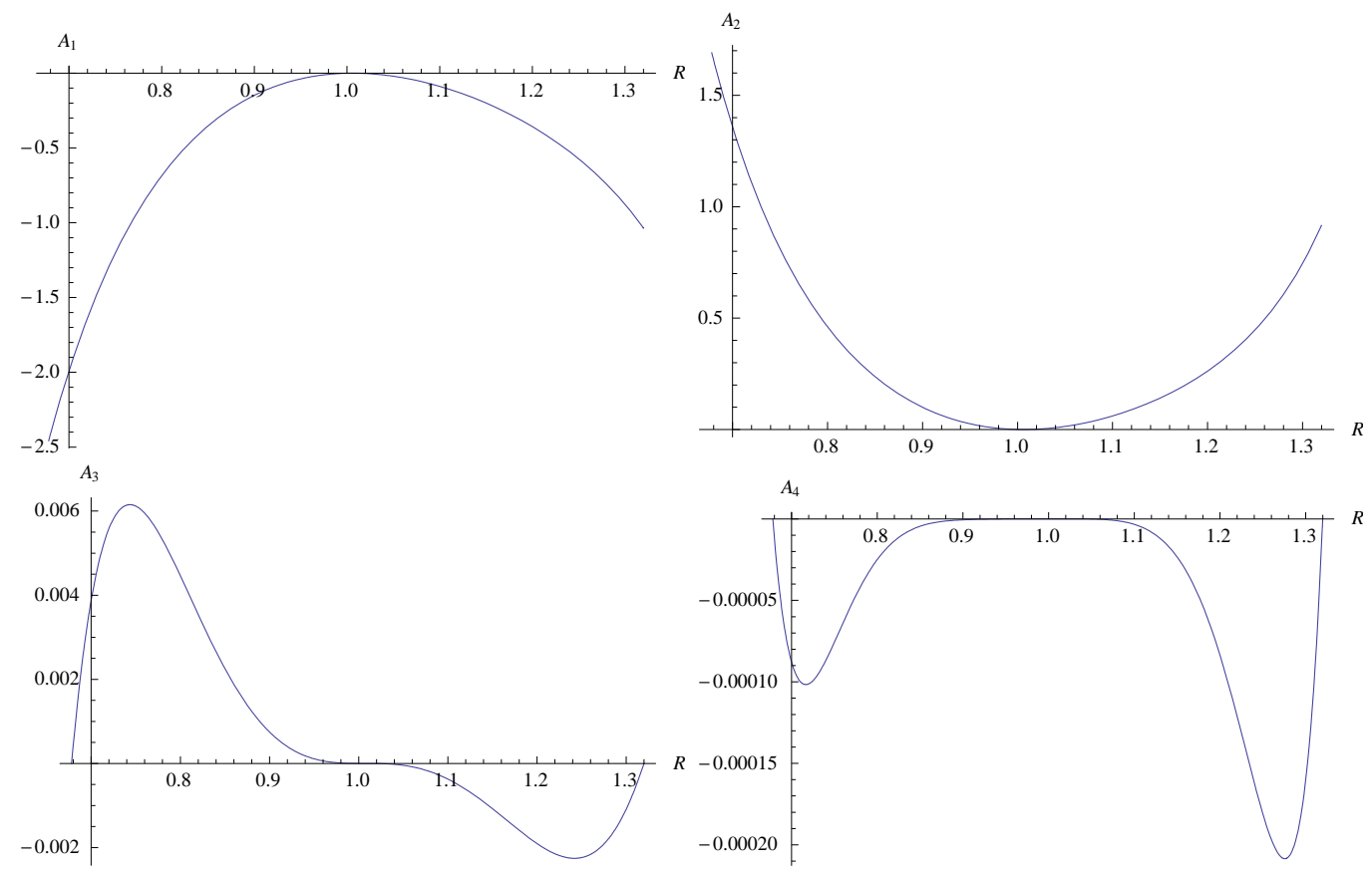

Figure 4: Profiles of the stability condition terms $A_{2}, A_{3}$ and $A_{4}$ on the plane $z=0$ for the Mach function (19) with $M_{a}^{2}=10^{-2}$.

but $A_{4}$ is destabilizing. Both flow contributions are at least three orders of magnitude lower than $A_{1}$. However taking into account the fact that the condition is sufficient, the above results do not necessarily imply that the equilibrium is unstable.

In summary, we have extended a Solovév-like equilibrium solution to plasmas with parallel incompressible flow. The advantageous characteristic of this solution is that it can contain an arbitrary number of free parameters which can be exploited to construct configurations with desirable boundary shaping and confinement figures of merit. In particular, by including in the solution fourteen free parameters we have derived ITER-like equilibria with prescribed values of the safety factor on axis and toroidal beta for both Mach-function profiles, peaked on and off axis. Application of a sufficient condition for linear stability showed that this condition is satisfied only in a small region irrespective of flow and flow shear unlike the cases of the nonlinear cat-eyes [12] and counter-rotating-vortices equilibria [13]. This result 
is in favor to the conjecture that the equilibrium nonlinearity may activate flow stabilization.

\section{Acknowledgements}

Part of this work was conducted during a visit of G.N.T. to the Max-PlanckInstitut für Plasmaphysik, Garching. The hospitality of that Institute is greatly appreciated.

This work was performed within the participation of the University of Ioannina in the Association Euratom-Hellenic Republic, which is supported in part by the European Union and by the General Secretariat of Research and Technology of Greece. The views and opinions expressed herein do not necessarily reflect those of the European Commission. 


\section{References}

[1] E. K. Maschke and H. Perrin, Plasma Physics 22, 579 (1980).

[2] E. Hameiri Phys. Fluids 26, 230 (1983).

[3] F. Bacciotti and C. Chiuderi, Phys. Fluids B 4, 35 (1992).

[4] H. Tasso and G. N. Throumoulopoulos, Phys. Plasmas 5, 2378 (1998).

[5] R. Betti and J. P. Freidberg, Phys. Plasmas 7, 2439 (2000).

[6] O. I. Bogoyavlenskij, Phys. Rev E 62, 8616 (2000).

[7] Ch. Simintzis, G. N. Throumoulopoulos, G. Pantis and H. Tasso, Phys. Plasmas 8, 2641 (2001).

[8] A. J. C. Belien, M. A. Botchev, J. P. Goedbloed, B. van der Holst, and R. Keppens, J. Comput. Phys. 182, 91 (2002).

[9] A. Thyagaraja and K. G. McClements, Phys. Plasmas 13, 062502 (2006).

[10] Roberto Antonio Clemente and Daniel Sterzo, Plasma Phys. Control. Fusion 51, 085011 (2009).

[11] D. Raburn and A. Fukuyama, Phys. Plasmas 17, 122504 (2010).

[12] G. N. Throumoulopoulos, H. Tasso and G. Poulipoulis, J. Phys. A: Math. Theor. 42, 335501 (2009).

[13] G. N. Throumoulopoulos and H. Tasso, Phys. Plasmas 17, 032508 (2010).

[14] L. S. Solovév, Sov. Phys.-JETP 26, 400 (1968).

[15] Antoine J. Cerfon and Jeffrey P. Freidberg, Phys. Plasmas 17, 032502 (2010).

[16] R. Srinivasan, L. L. Lao and M. S. Chu, Plasma Phys. Control. Fusion 52, 035007 (2010).

[17] G. N. Throumoulopoulos and H. Tasso, Phys. Plasmas 14, 122104 (2007).

[18] H. Tasso and G. N. Throumoulopoulos, J. Plasma Physics 78, 12012. 